\title{
The Operational Multiple Dosing Half-life: A Key to Defining Drug Accumulation in Patients and to Designing Extended Release Dosage Forms
}

\author{
Selma Sahin ${ }^{1,2}$ and Leslie Z. Benet ${ }^{1,3}$
}

Received September 19 2008; accepted November 4 2008; published online November 18, 2008

\begin{abstract}
Half-life $\left(t_{1 / 2}\right)$ is the oldest but least well understood pharmacokinetic parameter, because most definitions are related to hypothetical 1-compartment body models that don't describe most drugs in humans. Alternatively, terminal half-life $\left(t_{1 / 2, z}\right)$ is utilized as the single defining $t_{1 / 2}$ for most drugs. However, accumulation at steady state may be markedly over predicted utilizing $t_{1 / 2}, z$. An apparent multiple dosing half-life $\left(t_{1 / 2}\right.$, app $)$ was determined from peak and trough steady-state ratios and found to be significantly less than reported terminal $t_{1 / 2} \mathrm{~s}$ for eight orally dosed drugs with $t_{1 / 2, \mathrm{z}}$ values longer than one day. We define a new parameter, "operational multiple dosing half-life" $\left(t_{1 / 2, \text { op }}\right)$, as equal to the dosing interval at steady-state where the maximum concentration at steady-state is twice the maximum concentration found for the first dose. We demonstrate for diazepam that the well-accepted concept that $t_{1 / 2, \mathrm{z}}$ representing the great majority of the AUC will govern accumulation can be incorrect. Using oral diazepam, we demonstrate that $t_{1 / 2}$, op is remarkably sensitive to the absorption $t_{1 / 2}$, even when this absorption $t_{1 / 2}$ is much less than $t_{1 / 2, \mathrm{z}}$, and describe the relevance of this in designing extended release dosage forms. The $t_{1 / 2}$, op is compared with previously proposed half-lives for predicting accumulation.
\end{abstract}

KEY WORDS: apparent half-life; effective half-life; half-life; operational multiple dosing half-life; terminal half-life.

\section{INTRODUCTION}

Half-life is the oldest pharmacokinetic parameter and all clinicians believe they understand its relevance. Here we point out that although the relevance may be understood, the actual value reported for many drugs is not the relevant halflife and that even today the appropriate method for determining the relevant half-life has not been adequately discussed in the literature. In a number of cases, well accepted and generally used approaches are just wrong.

Half-life is the time interval in which half of the drug in a system is lost (when drugs do not exhibit saturable or any other nonlinear, anomalous or fractal kinetics, which is the condition considered in this work). If a drug is dosed at a frequency equal to the drug's half-life, the patient exposure to the drug at steady state during a dosing interval will be twice

\footnotetext{
${ }^{1}$ Department of Biopharmaceutical Sciences, University of California, 533 Parnassus Avenue, Room U-68, San Francisco, California 941430912, USA.

${ }^{2}$ Faculty of Pharmacy, Hacettepe University, 06100, Ankara, Turkey.

${ }^{3}$ To whom correspondence should be addressed. (e-mail: leslie. benet@ucsf.edu)

ABBREVIATIONS: AUC, area under the curve; AUMC, area under the moment curve; EHL, effective half-life; MRT, mean residence time in the body; $\mathrm{MRT}_{\mathrm{c}}$, mean residence time in the central compartment; $\mathrm{R}_{\mathrm{c}}$, Wagner's drug accumulation index; sd, single dose; ss, steady-state.
}

the exposure for a single first dose. If the dosing interval is less than the half-life then the exposure will be greater than double and if the dosing interval is greater than the half-life exposure will be less than double. But because drugs readily distribute out of and into the measured systemic circulation (blood/plasma/serum) from tissue compartments following a single dose all drugs will exhibit more than one half-life parameter. Thus, a problem arises when clinicians pick the half-life upon which to make exposure accumulation predictions, since drugs exhibit multiple half-lives. Usually the terminal (longest) half-life is chosen, but frequently this is incorrect. This work addresses this issue, which surprisingly has not been adequately explicated, considering the clinical significance of accumulation reaching either toxic or ineffective concentrations and its implication for designing extended release dosage forms.

It is well recognized that clearance divided by bioavailability defines the appropriate multiple dosing rate for a particular drug, i.e., the mass of drug dosed per unit time. For a drug following linear kinetics, this multiple dosing rate will yield the same average systemic concentration, that is, the same systemic exposure, no matter what the dosing interval. For example, at steady state a $240 \mathrm{mg}$ daily dose will yield the same average systemic concentration/exposure as $120 \mathrm{mg}$ every 12 h, $80 \mathrm{mg}$ every 8 h, $60 \mathrm{mg}$ every 6 h or $40 \mathrm{mg}$ every $4 \mathrm{~h}$, and this average systemic concentration/exposure will be equivalent to the steady state concentration/exposure obtained with a zero-order infusion of $10 \mathrm{mg} / \mathrm{h}$. The decision as to the appropriate dosing interval for that drug will depend 
on the half-life of the drug. Unfortunately, for most drugs there is no single half-life that can be readily accepted. This is due to fact that the systemic concentration time profile for most drugs is best described by a multiexponential function, thereby yielding more than one half-life to describe the drug. The choice of a single appropriate half-life value for such drugs is unclear. Often, the half-life describing the terminal log-linear phase of the log concentration time profile is selected as the single value to be reported. However, this terminal half-life may only represent a small fraction of the total clearance of a drug and, thus, is relatively unimportant in defining the accumulation of systemic concentrations upon multiple dosing. This uncertainty may be even further exacerbated when the drug is given orally and an absorption rate constant must be considered.

Probably the best example relating to the discrepancy between the terminal half-life and the operational half-life is for the commonly used anxiolytic drug diazepam, although to our knowledge this has not been examined carefully in the literature. If one looks up the diazepam half-life in Goodman and Gilman (43 \pm 13 h) (1), the Drug Information Handbook $(20-50 \mathrm{~h})(2)$ or any other standard reference book, a long half-life is reported. Yet the recommended dosing interval for diazepam is three to four times a day. Thus, one might suspect that the generally reported long half-lives are not relevant in achieving therapeutic efficacy for diazepam (we note that the package insert for diazepam tablets makes no mention of half-life).

Since, half-life is most important in defining multiple dosing regimens, it may be useful to describe parameters that expressly address this question. For a simple one compartment body model, there is only one half-life. Thus, if one were to dose the drug intravenously at a dosing interval equal to the half-life, systemic concentrations at steady-state would drop $50 \%$ during each dosing interval and accumulation would yield a maximum systemic concentration at steadystate that is twice the maximum systemic concentration found after the first dose. Thus for any drug following multicompartment linear drug disposition, we define the operational multiple dosing half-life as equal to the dosing interval at steady-state where the maximum concentration at steadystate is twice the maximum concentration found for the first dose and where the fall off to the trough plasma/blood concentration from the maximum plasma/blood concentration at steady-state is consistent with this half-life.

\section{Theory}

Various approximation equations have been proposed for calculating the dosing interval. Benet (3), as reported by Wagner (4), proposed the use of a dosing interval based on a systemic concentration multiple-dosing half-life $\left(t_{1 / 2, \mathrm{md}}\right)$ obtained by weighting individual half lives by their corresponding fractional area under curve following an intravenous bolus dose.

$$
\frac{1}{t_{1 / 2, m d}}=\sum_{i=1}^{n} \frac{f_{A U C, i}}{t_{1 / 2, i}}
$$

where $n$ is the number of exponential terms $(\lambda i)$ and coefficients ( $\mathrm{Li}$ ) describing the systemic concentration time curve and $f_{\mathrm{AUC}, \mathrm{i}}$ is the fraction of the area under the curve related to each half-life.

$$
f_{A U C, i}=\frac{L i / \lambda i}{\sum_{i=1}^{n} L i / \lambda i}
$$

For drugs, exhibiting multicompartment kinetics, the amount of drug in the body does not parallel systemic concentrations as is the case for a one compartment body model. Therefore, a different dosing interval must be chosen to describe the fall off of the amount of drug in the body during a dosage interval at steady-state. Such a multiple dosing half-life for amount of drug in the body $\left(t_{1 / 2, \mathrm{~A}}\right)$ may also be defined:

$$
t_{1 / 2, A}=\sum_{i=1}^{n} f_{A U C, i} \cdot t_{1 / 2, i}
$$

Both of these multiple dosing half-lives can be defined in terms of well known pharmacokinetic parameters.

$$
\begin{gathered}
t_{1 / 2, m d}=\frac{\ln (2)}{k_{10}}=\ln (2) \cdot \frac{V_{1}}{C L} \\
t_{1 / 2, A}=\ln (2) \cdot M R T=\ln (2) \cdot \frac{V_{s s}}{C L}
\end{gathered}
$$

where $k_{10}$ is the elimination rate constant from the central compartment in a mammillary model where elimination occurs only from the central compartment; $V_{1}$ is the volume of the central compartment; $\mathrm{CL}$ is clearance; MRT is mean residence time in the body (following an intravenous bolus dose) and $V_{\mathrm{ss}}$ is the volume of distribution at steady state. Note that $t_{1 / 2 \mathrm{~A}}$ can be determined by non-compartmental models, while $t_{1 / 2 \text {, md }}$ requires a fit of a polyexponential equation to the data to be able to determine $V_{1}$.

Wagner (4) approached the problem in the context of mean residence time principles. He proposed that a suitable dosage interval can be estimated by a factor times the sum of mean transit time of the central compartment and the mean transit time of the absorption site. Such a dosing interval provides a ratio, the maximum steady-state plasma concentration/minimum steady-state plasma concentration, which averages $\sim 2$.

Veng-Pedersen and Modi (5) presented an explicit formula for simple calculations of the dosing time that results in a steady-state peak-to-trough ratio of 2 for extravascular administration described by a two-exponential expression (i.e., one compartment body model with first order absorption). However, when more than two exponential terms are needed in the approximation of the drug level profile from the extravascular administration, iterative calculation of the dosing interval using a computer was suggested.

These various approximation equations recommended for estimation of dosing interval are summarized in Table I, where MRTc is the mean residence time in the central compartment and MIT is the mean input time.

The problem has also been addressed by Kwan et al. (6) and Boxenbaum and Battle (7) who proposed the concept of an "effective half-life" (EHL) utilizing Wagner's (8) drug accumulation index $\left(R_{\mathrm{c}}\right)$ :

$$
R_{c}=A U C_{s s, 0 \rightarrow \tau} / A U C_{s d, 0 \rightarrow \tau}=A U C_{s d, 0 \rightarrow \infty} / A U C_{s d, 0 \rightarrow \tau}
$$


where $A U C_{s s, 0 \rightarrow \tau}$ is the area under the curve at steady-state (ss) during a dosing interval, $\tau, A U C_{s d, 0 \rightarrow \tau}$ is the area under the curve for a single dose (sd) during the same time interval, $\tau$, i.e., from time 0 to time $\tau$, while $A U C_{s d, 0 \rightarrow \infty}$ is the AUC for a single dose over all time, which for drugs following linear kinetics is equal to $A U C_{s s, 0 \rightarrow \tau}$. These authors then calculate the EHL from $\mathbf{R}_{\mathrm{c}}$ and $\tau$ assuming the drug follows onecompartment i.v. bolus dose kinetics at steady-state as per Eq. 7:

$$
E H L=\ln 2 \tau / \ln \left[\left(R_{c}-1\right) / R_{c}\right]
$$

Note in this relationship that the EHL is a function of the dosing interval, as opposed to being only a drug related value.

The EHL concept was developed by Kwan et al. (6) and Colburn (9) because they were unable to measure a relevant terminal half-life for NSAIDs that exhibited biliary cycling. Another half-life term, the "functional half-life" was introduced by Hsu et al. (10) to allow a simple half-life calculation at steady-state for a drug exhibiting nonlinear kinetics, ritonavir. This half-life has generally been designated by others as the "apparent multiple dosing half-life" $\left(t_{1 / 2, \text { app }}\right)$, the terminology we use here. It is calculated using Eq. 8 at steady-state from the peak-to-trough ratios and the time interval $(\Delta t)$ between the measured concentrations, which will always be less than the dosing interval $(\tau)$ for oral dosing:

$$
\frac{C_{\max , s s}}{C_{\min , s s}}=\exp \left(0.693 \cdot \Delta t / t_{1 / 2, a p p}\right)
$$

Like the EHL, $t_{1 / 2 \text {,app }}$ will also be a function of the dosing interval, as opposed to being only a drug related value. The various half-life terms are defined in Table II in terms of the methods of determination. The last column in Table II provides comments on the usefulness of each term with respect to predicting drug accumulation upon multiple dosing as will be discussed subsequently.

\section{METHODS AND RESULTS}

The literature was reviewed for drugs with reported terminal half lives longer than one day where for orally dosed drugs peak and trough measurements at steady state were available. An "apparent multiple dosing half life" ( $\left.t_{1 / 2, \text { app }}\right)$ was determined using Eq. 8. These results are presented in Table III. All of the apparent steady-state half-life values are less than $55 \%(15.3-54.3 \%)$ of the reported terminal half-lives.
Because of the discrepancy between recommended dosing interval and terminal half-life for diazepam discussed above, we chose this drug for simulation. Dhillon and Richens (19) used a 2-compartment body model to describe the time course of a $10 \mathrm{mg}$ i.v. diazepam dose in six healthy volunteers. The equation describing the concentration $(\mathrm{C}$ in $\mathrm{mg} / \mathrm{L})-$ time ( $t$ in hours) data is:

$$
C_{i v}=1207 e^{-3.2 t}+183 e^{-0.0233 t}
$$

In terms of Eq. 2, where for diazepam $L_{1}=1207 \mathrm{mg} / \mathrm{L}, L_{2}=$ $183 \mathrm{mg} / \mathrm{L}, \lambda_{1}=3.2 \mathrm{~h}^{-1}$ and $\lambda_{2}=0.0233 \mathrm{~h}^{-1}$, it can be readily calculated that the fraction of AUC related to the terminal $29.7 \mathrm{~h}$ half-life $(\ln 2 / 0.0233)$ accounts for the great majority $(95.4 \%)$ of the area. We calculated the operational multiple dosing half-life $\left(t_{1 / 2, \text { op }}\right)$ by iteration of Eq. 9 . The three values for each method (iteration, Wagner equation, Benet equation), the $t_{1 / 2, \mathrm{~A}}$ calculated from AUMC/AUC and the EHL calculated at $\tau=t_{1 / 2 \text {,op }}$ are given in Table IVA. Both the equations of Benet (3) and Wagner (4) underestimate the dosing interval that would result in a two fold increase in $C_{\max }$ upon multiple dosing, which corresponded to a $C_{\max } /$ $C_{\text {min }}$ ratio of 2.0 at steady-state. In contrast, the EHL and $t_{1 / 2, \mathrm{~A}}$ markedly overestimate the operational multiple dosing half-life. Following intravenous dosing $t_{1 / 2 \text {,app }}$ will equal $t_{1 / 2 \text {,op }}$ when $\tau=t_{1 / 2, \text { op }}$, but will change as a function of $\tau$ as noted in Table II and shown subsequently.

However in most cases diazepam is given orally. The study of Kaplan et al. (14) included a $10 \mathrm{mg}$ oral dose in four healthy volunteers yielding a mean absorption half-life of $0.36 \mathrm{~h}\left(k_{\mathrm{a}}=1.92 \mathrm{~h}^{-1}\right)$. We carried out simulations to determine the operational half-life using this absorption rate constant as given in Eq. 10:

$$
C_{\text {oral }}=-1810 e^{-3.2 t}+185 e^{-0.0233 t}+1625 e^{-1.92 t}
$$

as well as for absorption half-lives of $0.25 \mathrm{~h}\left(k_{\mathrm{a}}=2.77 \mathrm{~h}^{-1}\right)$ and $2 \mathrm{~h}\left(k_{\mathrm{a}}=0.347 \mathrm{~h}^{-1}\right)$. The iterative operational half-life was determined when $C_{\max , s \mathrm{~s}} / C_{\mathrm{max}, \mathrm{sd}}=2.0$. As opposed to the intravenous bolus dose, this operational half-life is close but does not correspond exactly to the maximum half-life calculated from the concentration at steady-state to the minimum concentration at steady-state, since $t_{\max }$ changes upon multiple dosing. These results compared to the values predicted by Wagner's and Benet's equations, the $t_{1 / 2, \mathrm{~A}}$ and the EHL when $\tau=t_{1 / 2 \text {,op }}$ are given in Table IVB. Again the Wagner and Benet equations underestimate the iterative

\begin{tabular}{|c|c|c|c|}
\hline \multicolumn{4}{|c|}{ Dosing interval $(\tau)$} \\
\hline Model & Benet (3) & Wagner (4) & Veng-Pedersen and Modi (5) \\
\hline IV-bolus, Multicompartment & $\begin{array}{l}0.693 \times \mathrm{MRTc} \\
=\frac{0.693}{k_{10}}\end{array}$ & $=\frac{0.75}{k_{10}}$ & NA \\
\hline $\begin{array}{l}\text { Extravascular administration, } \\
\text { One compartment }\end{array}$ & $\begin{array}{l}0.693 \times(\mathrm{MRT}+\mathrm{MIT}) \\
=\frac{1}{k_{10}}+\frac{1}{k_{a}}\end{array}$ & $=\frac{1}{k_{10}}+\frac{1}{k_{a}}$ & $\begin{array}{l}=\left[1.78 e^{-0.739 \ln (\beta 2 / \beta 1)}+0.684\right] / \beta 1 \text { for } 1 \leq \beta 2 / \beta 1 \leq 1000 \\
=\ln (2) / \beta 1 \text { for } \beta 2 / \beta 1>1,000 \text { where } \beta 2 \text { is the faster rate constant }\end{array}$ \\
\hline $\begin{array}{l}\text { Extravascular administration, } \\
\text { Multicompartment }\end{array}$ & $=\frac{10}{k_{10}}+\frac{k_{q}}{k_{a}}$ & $=1.35\left(\frac{1}{k_{10}}+\frac{1}{k_{a}}\right)$ & Calculate iteratively on a computer \\
\hline
\end{tabular}
solutions, with the discrepancies markedly increasing as the absorption rate decreases. In contrast, the EHL and $t_{1 / 2, \mathrm{~A}}$ are

Table I. Recommended Dosing Intervals 

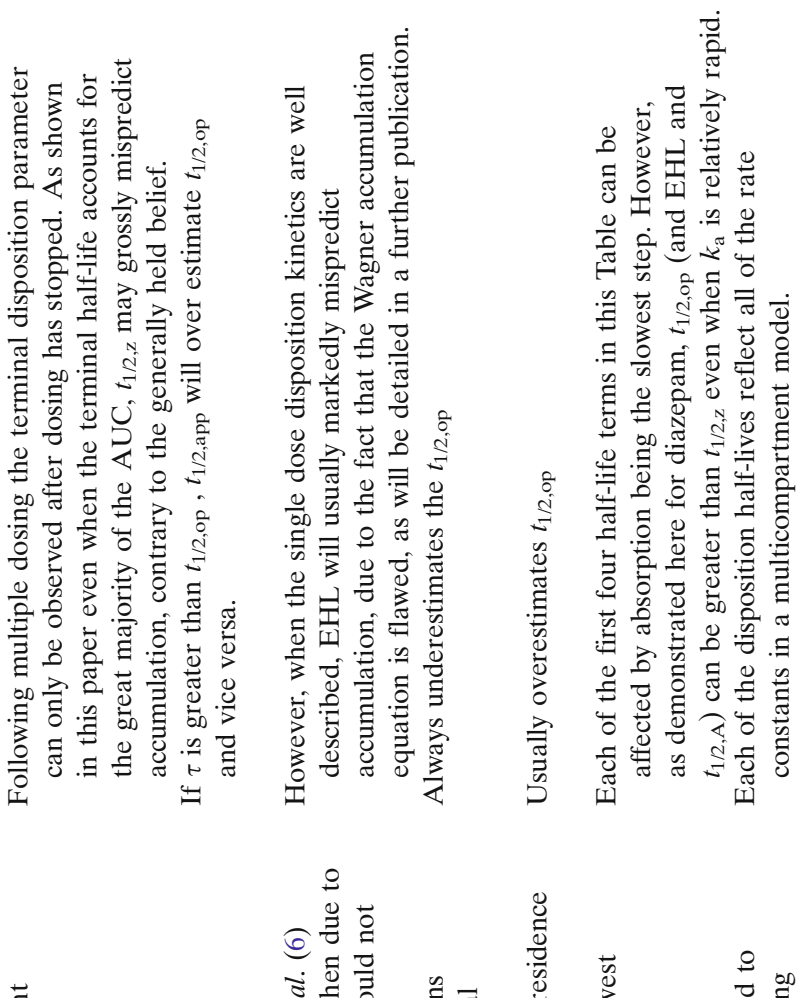

$\stackrel{\circ}{\circ}$

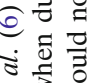

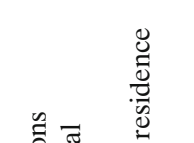

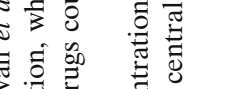

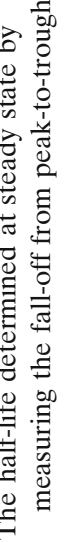
各节范 ङ

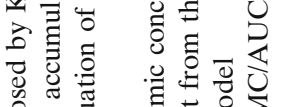

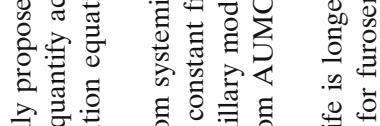

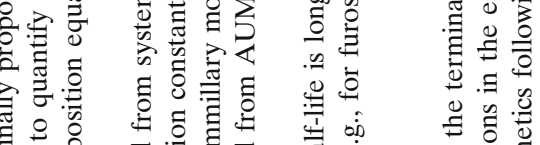

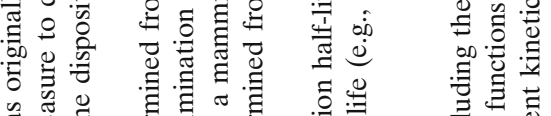

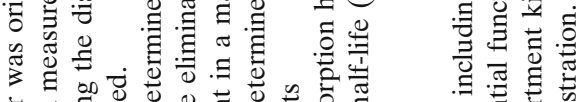

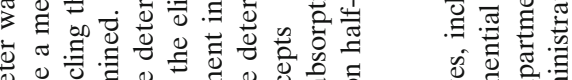

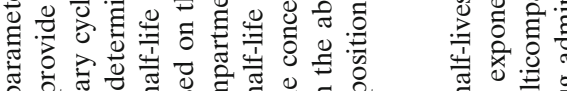

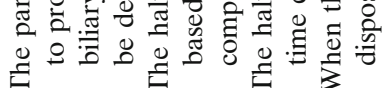

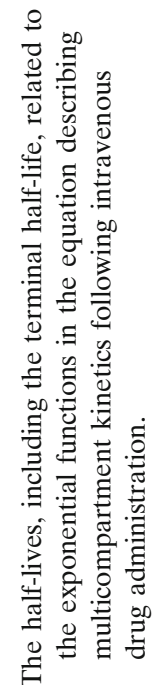

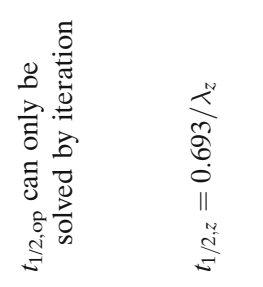

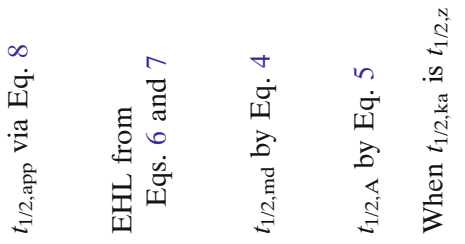

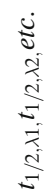

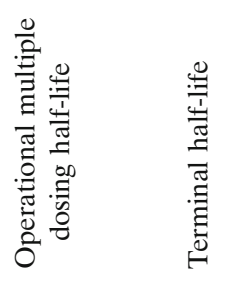

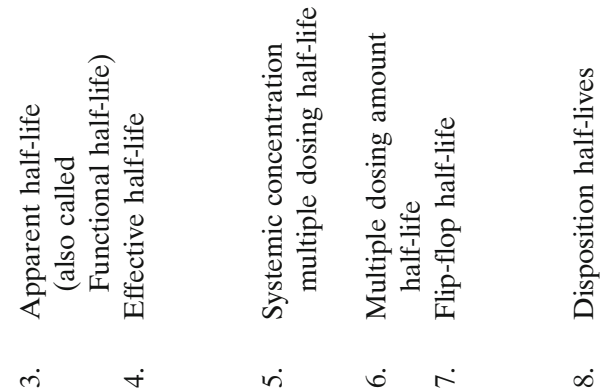


Table III. Terminal Half-life $v s$ Apparent Steady-state Half-life Calculated by Eq. 8

\begin{tabular}{lcccl}
\hline Compound & Route and dosing interval & Terminal $t_{1 / 2, \mathrm{z}}(\mathrm{h})$ & Apparent steady-state $t_{1 / 2, \text { app }}(\mathrm{h})$ & Reference \\
\hline Bepridil & p.o., $24 \mathrm{~h}$ & 42 & 10.6 & Benet $(11)$ \\
Chloroquine & p.o. 1 day & $7-10$ days & 1.6 days & Augustijns et al. $(12)$ \\
Chlorthalidone & p.o., $24 \mathrm{~h}$ & 64.8 & 24.5 & Colleste et al. $(13)$ \\
Diazepam & p.o., $24 \mathrm{~h}$ & 30.2 & 16.4 & Kaplan et al. $(14)$ \\
Everolimus & p.o., $24 \mathrm{~h}$ & 11.0 & Budde et al. $(15)$ \\
LAAM & p.o., $48 \mathrm{~h}$ & 32 & 19.8 & Newcombe et al. (16) \\
Rifabutin & p.o., $24 \mathrm{~h}$ & 46.8 & 9.67 & Li et al. (17) \\
Sirolimus & p.o., $12 \mathrm{~h}$ & 53.3 & 9.5 & Zimmerman and Khan (18) \\
\hline
\end{tabular}

${ }^{a}$ Levo-alpha acetyl methadol

not very sensitive to $k_{\mathrm{a}}$, markedly overestimating the accumulation half-life for the rapid $k_{\mathrm{a}}$ values, but as can be seen in Table IVB at some $k_{\mathrm{a}}$ between 1.92 and $0.347 \mathrm{~h}^{-1}$, each will equal $t_{1 / 2, \mathrm{op}}$.

Simulations were carried out to elucidate the role of the various usual dosing intervals on the maximum to minimum plasma concentration ratio at steady-state, as well as the ratio of the maximum concentration at steady-state $\left(C_{\text {max,ss }}\right)$ to the maximum concentration for the single dose $\left(C_{\text {max,sd }}\right)$. The dosing schedules simulated were once a day $(\tau=24 \mathrm{~h})$, twice a day $(\tau=12 \mathrm{~h})$, three times a day $(\tau=8 \mathrm{~h})$ or six times a day $(\tau=$ $4 \mathrm{~h}$ ). Time course for the simulations were set to $168 \mathrm{~h}$ for i.v. and $264 \mathrm{~h}$ for oral dosings and data generated every $0.125 \mathrm{~h}$. All simulations were performed for 2-(oral and i.v.) compartment body models using WinNonlin (Version 2.1).

Figure $1 \mathrm{~A}$ depicts the ratio $C_{\mathrm{max}, \mathrm{ss}} / C_{\mathrm{min}, \mathrm{ss}}$ and the ratio of maximum concentration at steady-state to the maximum concentration for a single dose $\left(C_{\text {max,ss }} / C_{\text {max,sd }}\right)$ calculated for the dosing intervals $4 \mathrm{~h}, 8 \mathrm{~h}, 12 \mathrm{~h}$ and $24 \mathrm{~h}$ for the twocompartment i.v. doses of diazepam. Note that the ratio of peak to trough at steady-state increases with dosing interval while the inverse is seen with ratio of maximum concentrations at steady-state to that for the first dose. The curves intersect at the operational half-life, which gives a ratio of 2.0. Fig. 1B depicts these two ratios as a function of dosing interval for the oral data using the Kaplan et al. (14) reported $k_{\mathrm{a}}=1.92$. It is obvious that the ratios in Fig. 1 are close to 2.0 when the dosing interval is on either side of the operational half-life, but that marked deviations occur at more distant dosing intervals.

\section{DISCUSSION}

Selection of the appropriate dosing interval is an important clinical decision for a drug in a patient population. This is especially critical for narrow therapeutic index drugs. Concern may be expressed that the pharmacodynamic halflife will be more relevant than the pharmacokinetic value. We will return to this issue at the end of the Discussion section. However, if a pharmacokinetic half-life is clinically relevant and if it is to be used to design and justify an extended release drug product, then the appropriate half-life must be selected. Yet, many clinicians and clinical scientists believe and have been taught that the terminal half-life is the value most useful in selecting the dosing interval. However, we are unaware of any test of this hypothesis. In Table III we list eight drugs with long terminal half-lives, which we believe exhibit linear kinetics at the doses studied, where data for multiple oral dosing allowed determination of an apparent half-life at steady-state. In each case this apparent half-life was less than $55 \%$ of the reported terminal half-life. For rifabutin and sirolimus the apparent half-life values were $15-18 \%$ of the terminal half-lives.

Why does a clinician want to know the half-life? We believe that the most clinically relevant use of this value is the ability to predict the accumulation of drug in a patient upon multiple dosing. If a patient were dosed at intervals equivalent to this operational half-life, one would expect accumulation to double and peak to trough ratios at steady-state would be related by this half-life. In essence one is attempting to find a single half-life for a drug exhibiting multicompartment

Table IV. Calculations of Operational Half-lives (hr) at Steady-state Expected for Peak Multiple Dose to Peak Single Dose Ratios of 2.0 Compared to the Methods of Wagner (4), Benet (3), the $t_{1 / 2, \mathrm{~A}}$ from MRT and the Effective Half-Life $(6,7)$ when $\tau=t_{1 / 2, \text { op }}$

\begin{tabular}{|c|c|c|c|c|c|}
\hline & $t_{1 / 2, \mathrm{op}}$ by iteration & $t_{1 / 2, \mathrm{md}}$ Wagner & $t_{1 / 2, \mathrm{md}}$ Benet & $t_{1 / 2, \mathrm{~A}}$ & EHL \\
\hline \multicolumn{6}{|c|}{$\begin{array}{l}\text { A. Intravenous data of Dhillon and Richens (19) } \\
\text { fit to a two-compartment body model }\end{array}$} \\
\hline & 5.30 & 4.44 & 4.10 & 28.4 & 21.6 \\
\hline \multicolumn{6}{|c|}{$\begin{array}{l}\text { B. Oral data using the two-compartment parameters } \\
\text { in Eq. } 9 \text { and the reported absorption } k_{\mathrm{a}}=1.92 \mathrm{~h}^{-1} \\
\text { of Kaplan et al. (14) and selected higher and lower values }\end{array}$} \\
\hline$k_{\mathrm{a}}=2.77 \mathrm{~h}^{-1}$ & 12.95 & 8.48 & 6.28 & 28.7 & 26.4 \\
\hline$k_{\mathrm{a}}=1.92 \mathrm{~h}^{-1}$ & 15.2 & 8.69 & 6.44 & 28.8 & 27.1 \\
\hline$k_{\mathrm{a}}=0.347 \mathrm{~h}^{-1}$ & 35.0 & 11.9 & 8.80 & 30.4 & 30.6 \\
\hline
\end{tabular}


A

2-COMP IV $\left(\mathrm{t}_{1 / 2, \mathrm{op}}=\mathbf{5 . 3 0 \mathrm { hr } )}\right.$

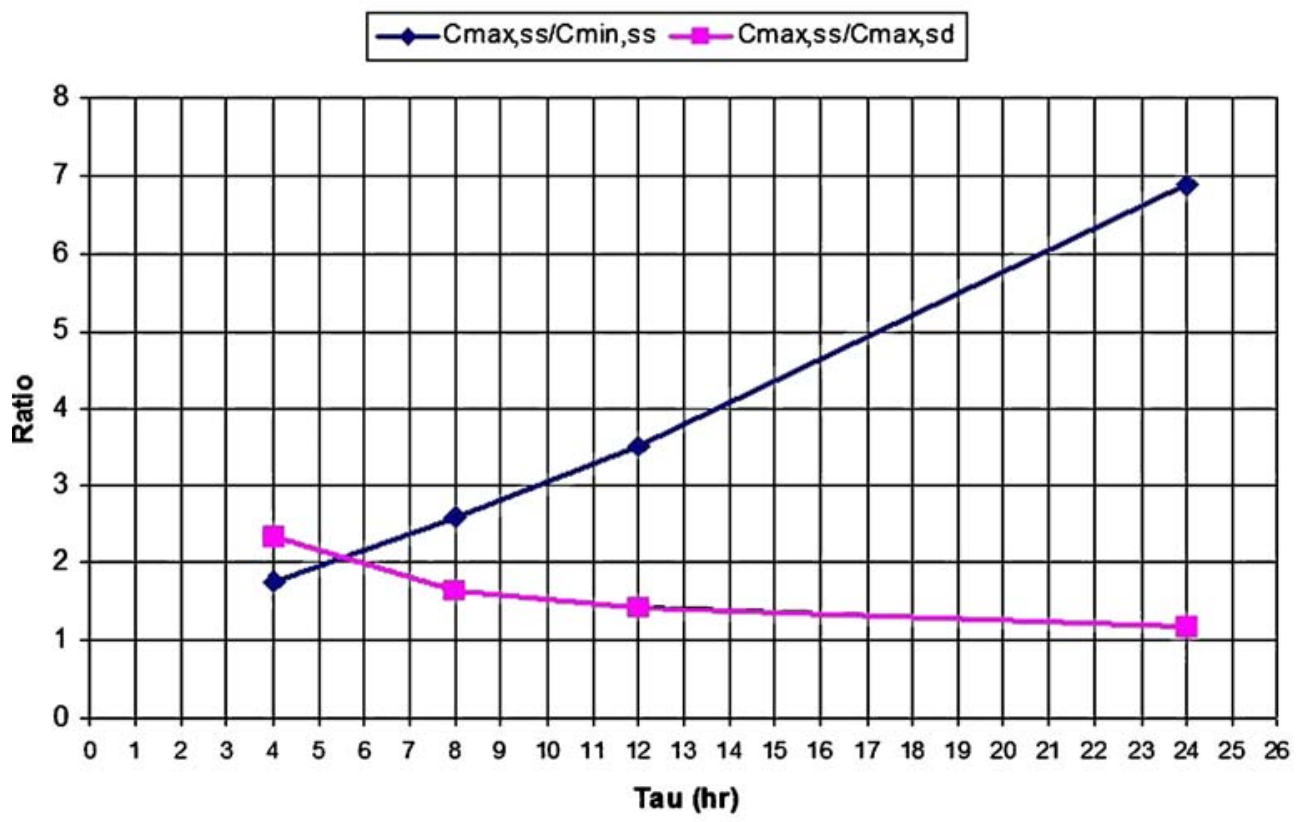

B

2-COMP ORAL ( $\left.\mathrm{t}_{12, \mathrm{op}}=15.2 \mathrm{hr}\right)$

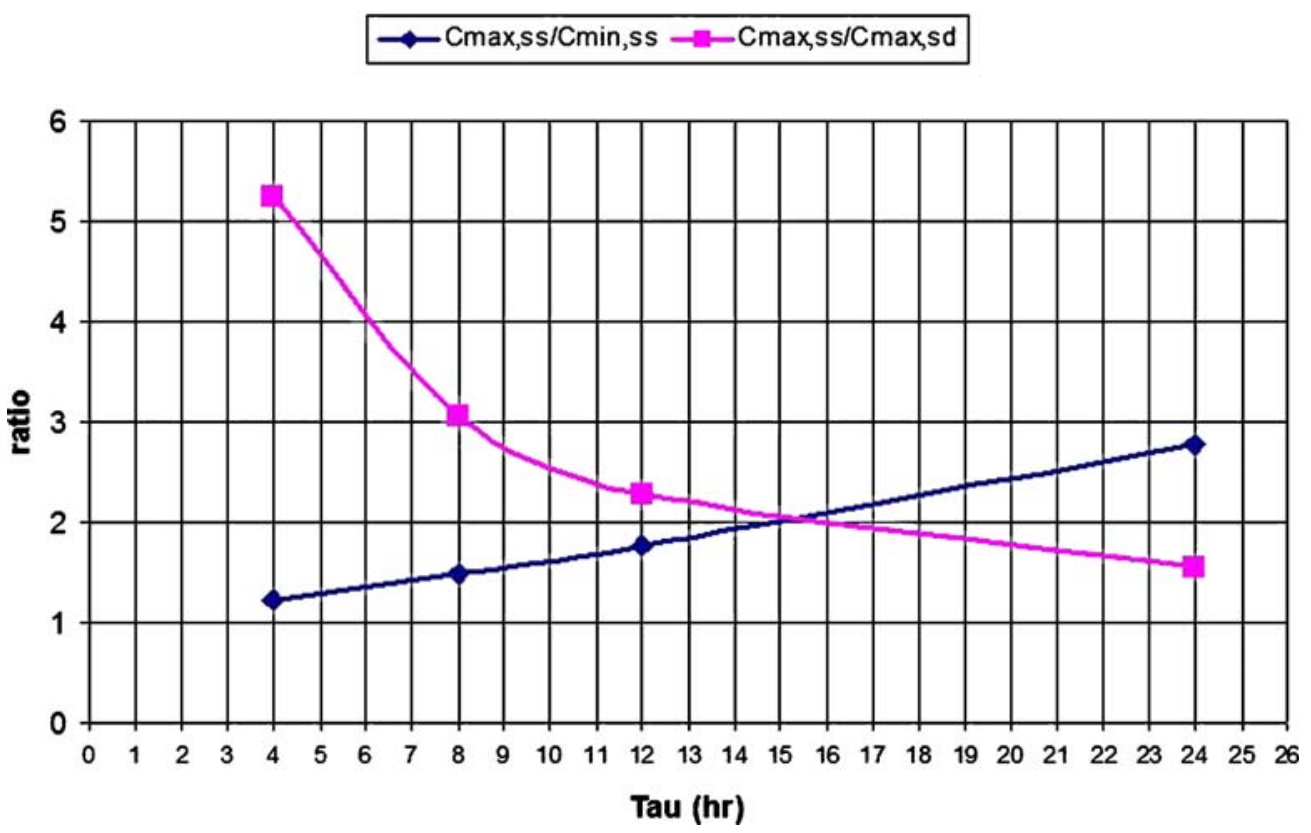

Fig. 1. A The ratio (black diamonds) of maximum to minimum concentration at steady-state $\left(C_{\mathrm{max}, \mathrm{ss}} / C_{\mathrm{min}, \mathrm{ss}}\right)$ and the ratio (grey squares) of maximum concentration at steady-state to the maximum concentration for a single dose $\left(C_{\max , s \mathrm{~s}} / C_{\max , \mathrm{sd}}\right)$ calculated for $4 \mathrm{~h}, 8 \mathrm{~h}, 12 \mathrm{~h}$ and $24 \mathrm{~h}$ dosing intervals for two-compartment i.v. doses of diazepam. B The ratio (black diamonds) of maximum to minimum concentration at steady-state $\left(C_{\mathrm{max}, \mathrm{ss}} / C_{\mathrm{min}, \mathrm{ss}}\right)$ and the ratio (grey squares) of maximum concentration at steady-state to the maximum concentration ratio for a single dose $\left(C_{\mathrm{max}, \mathrm{ss}} / C_{\mathrm{max}, \mathrm{sd}}\right)$ calculated for $4 \mathrm{~h}, 8 \mathrm{~h}, 12 \mathrm{~h}$ and $24 \mathrm{~h}$ dosing intervals for two-compartment oral data using $k_{\mathrm{a}}$ value of $1.92 \mathrm{~h}^{-1}(14)$.

kinetics that at steady-state approximates what one would expect for a drug described by a 1-compartment body model. Benet $(3,4)$ first addressed this problem in 1985 suggesting that 0.693 multiplied by the mean residence time in the central compartment $\left(\mathrm{MRT}_{\mathrm{c}}\right)$ would predict the half-life. We used simulations using two compartment analyses of diazepam to evaluate the predictability. As can be seen in
Table IVA the predictions using Benet's approach for i.v. diazepam dosing underestimate the iteratively determined actual values. But the Benet predictions yield a much better estimate than the terminal half-life, which is $29.7 \mathrm{~h}$ from the 2compartment data fit and the EHL and $t_{1 / 2, \mathrm{~A}}$. Wagner, in referencing the Benet equations, realized that the values were underestimated and added a scaling factor, which here for 
diazepam does yield better, but still underestimated predictions. In contrast, the EHL and $t_{1 / 2, \mathrm{~A}}$ markedly overestimate the operational half-life. From the $5.30 \mathrm{~h}$ operational half-life given in Table IVA one can understand the proposed recommended diazepam dosing of three to four times a day, although the source of this labeling recommendation is unknown to us.

The most surprising finding in this work is the sensitivity of the operational half-life to the absorption rate constant following oral dosing. For example, even for a very rapid absorption $\left(t_{1 / 2}=0.25 \mathrm{~h}\right)$ the operational half-life following oral dosing increases by $144 \%$ from that found for i.v. dosing (compare values for $k_{\mathrm{a}}=2.77 \mathrm{~h}^{-1}$ in Table IVB with values in IVA). Note that for an absorption half-life of $2 \mathrm{~h}\left(k_{\mathrm{a}}=0.347 \mathrm{~h}^{-1}\right)$ in the two-compartment model, the operational half-life $(35.0 \mathrm{~h})$ is larger than the terminal half-life $(29.7 \mathrm{~h})$. This sensitivity of the operational half-life to the absorption rate constant probably explains the frequently reported differences in peak to trough ratios noted between morning and evening dosing for twice daily drug regimens. One would expect differences in absorption rate for the dose taken at bed time versus that taken in the morning. It also explains why peak to trough ratio across a population and even within an individual are more variable than average concentrations, since the former is dependent on $k_{\mathrm{a}}$ and the latter on clearance.

Reviewers of drafts of this manuscript were concerned that diazepam may be unique and that other drugs may not exhibit this marked change in $t_{1 / 2 \text {,op }}$ with relatively small changes in the absorption rate constant. In a following manuscript we will show that two further drugs in Table III, everolimus and bepridil, also show significant changes in $t_{1 / 2, \text { op }}$ as a function of $k_{\mathrm{a}}$.

The relationships depicted in Fig. 1 provide some useful insights. At dosing intervals greater than $t_{1 / 2 \text {,op }}$ calculating a half-life from $C_{\text {max,ss }} / C_{\text {min,ss }}$ will overestimate $t_{1 / 2 \text {,op }}$, while calculating a half-life from $\mathrm{C}_{\text {max,ss }} / \mathrm{C}_{\text {max,sd }}$ will underestimate

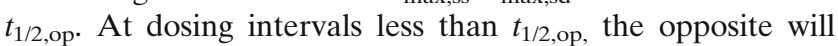
occur. Looking back at Table III we can thus suggest that the apparent steady-state half-lives for all drugs, except chloroquine and chlorthalidone, overestimate $t_{1 / 2, \mathrm{op}}$, and chlorthalidone is well estimated, since $t_{1 / 2 \text {,op }}$ approximates the dosing interval. The value for sirolimus, in this study where the patients also received concomitant cyclosporine, is probably also well estimated. That the value for diazepam is overestimated in Table III is confirmed since the calculated $t_{1 / 2 \text {,op }}$ for diazepam using the pharmacokinetic parameters from that study can be seen in Table IV.

Reviewing the recent literature provides a confirming example for the principles presented here. Iwamoto et al. (20) evaluated the accumulation of the HIV integrase inhibitor raltegravir following single and multiple twice daily 100,200 , 400,600 and $800 \mathrm{mg}$ oral doses. The average $C_{\text {max,ss }} / C_{\text {max,sd }}$ was 1.0, indicating no accumulation. They also compared areas under the curve at steady-state to areas under the curve for the same time interval $(12 \mathrm{~h})$ for the first dose and reported an average accumulation of 1.06 (This ratio substituted into Eq. 7 indicates an effective half-life of $2.90 \mathrm{~h}$ ). Yet, Iwamoto et al. (20) report mean terminal half-lives for the 5 different doses that range from 9.9 to $12.1 \mathrm{~h}$. It is obvious that the $t_{1 / 2, \text { op }}$ for raltegravir must be significantly shorter if no accumulation is observed, as here also shown for the EHL calculation.
However, an even more potentially useful application is apparent from the results in Table IVB. Today, the general belief in the development of extended release dosage forms is that drug release from the dosage form must be the rate controlling step. This is often difficult to achieve because of gastrointestinal transit time and poor absorption from the ileum and colon. Yet, the results in Table IVB suggest, that at least for some drugs, a relatively modest change in the absorption rate (by altering drug release from the dosage form) can markedly change the operational half-life. Drug product formulators avoid changing a drug's disposition kinetics, since this would involve inhibiting, inducing or activating enzymes and transporters that certainly would generate regulatory concerns; furthermore as mentioned above creating drug delivery devices that control the rate of oral absorption and make it the rate limiting step is difficult to achieve and when achieved frequently lead to patent protection that precludes others from using a similar approach. However, making relatively small changes in absorption are easily within the expertise of drug product formulators, and because such changes have frequently been employed in the past, such as adding a shellac coating to micro/nano-particles, little patent protection would be afforded. Thus, we now recognize that for drugs where single dose kinetics has been accurately quantitated, simulations may be easily carried out where the absorption rate is modified to determine the sensitivity of the multiple dosing operational half-life to these changes as we have shown for diazepam in Table IVB. The procedure is as follows:

1. A pharmacokinetic compartment model is fit to the i.v. data, or more likely the oral data, in humans yielding equations such as Eqs. 9 and 10 for diazepam. Test for linearity by evaluating different doses or multiple dosing.

2. Calculate the pharmacokinetic parameters that describe the single dose data. If only oral dosing data is available, distinguish the absorption rate constant from the disposition parameters by deliberately altering absorption rate as described by Boni et al. (21) or using an intercept method (22).

3. Choose the desired dosing interval, $\tau$.

4. Modify the equation describing single dose oral data to a multiple dosing equation at steady-state by multiplying each exponential function by the appropriate multiple dosing function, $1 /\left[1-\exp \left(-\lambda_{i} \times \tau\right)\right]$ and changing the time function in each exponential term to t', the time within a dosing interval, as first presented by Dost (23) and implemented by Benet (24) for his general treatment of mammillary models. For Eq. 10, with a $k_{\mathrm{a}}=1.92 \mathrm{~h}^{-1}$, this would result in the following Eq. 11:

$$
\begin{aligned}
C_{\text {oral }}= & -\frac{1810}{1-e^{-3.2 \tau}} e^{-3.2 t^{\prime}}+\frac{185}{1-e^{-0.0233 \tau}} e^{-0.0233 t^{\prime}} \\
& +\frac{1625}{1-e^{-k_{a} \tau}} e^{-k_{a} t^{\prime}}
\end{aligned}
$$

5. Iterate with different values of $k_{\mathrm{a}}$ to determine which $k_{\text {a }}$ value will achieve an operational half-life that equals the desired $\tau$. Note that all of the coefficients in Eqs. 10 and 11 contain $k_{\mathrm{a}}$ in the numerator and 
denominator (24). This step will always be successful, but for certain drugs and selected dosing intervals the solution may indicate that only a dosage form where absorption is the slowest, rate controlling step, will succeed. Then perhaps a shorter $\tau$ may be tested. However, for many drugs we believe that readily achievable absorption halflives, that are less than the terminal half-life, will suggest that extended release formulations will be successful.

What are the take home messages from this analysis?

1. Predicting a dosing interval for intravenous bolus dosing accumulation at steady-state can be reasonably obtained by $0.693 \mathrm{MRTc}$, although this will be an underestimate. The terminal half-life will be a marked over prediction of the appropriate dosing interval.

2. Even for drugs where the terminal half-life represents the great majority of the AUC (e.g., diazepam >95\%), $t_{1 / 2 \text {,op }}$ may be much smaller for both i.v. and oral dosing.

3. Following oral dosing, even for very fast absorption, $t_{1 / 2 \text {,op }}$ may be significantly greater than that for i.v. dosing. The $t_{1 / 2, \text { op }}$ value may be quite sensitive to changes in $k_{\mathrm{a}}$. In some cases, with slow absorption, $t_{1 / 2}$, op can be greater than the terminal half-life. But this is not restricted to flip-flop models where $k_{\mathrm{a}}<\lambda_{\mathrm{z}}$ (where $\lambda_{\mathrm{z}}$ is the terminal disposition constant following i.v. dosing). Here for diazepam in Table IVB, $t_{1 / 2, \mathrm{op}}>t_{1 / 2, \mathrm{z}}$ when the absorption half life was only $2 \mathrm{~h} v s t_{1 / 2, \mathrm{z}}=$ $29 \mathrm{~h}$ and $t_{1 / 2, \mathrm{op}}=35 \mathrm{~h}$

4. For orally dosed drugs, it is not possible to simply predict $t_{1 / 2, \text { op }}$; this can only be done by iterative calculations, as first suggested by Veng-Pedersen and Modi (5).

5. The terminal half-life only describes drug loss from the body after drug dosing has stopped; for a number of drugs, it is not a good predictor of accumulation at steady-state, or of the time course of drug fall off during a dosing interval at steady-state, even when the terminal half-life relates to the majority of the AUC.

6. The difficulty pointed out here for predicting accumulation is most significant for drugs exhibiting long terminal half-lives as given in Table III. Frequently, for drugs with half-lives of $12 \mathrm{~h}$ or less, in our experience, accumulation prediction error will not be significant. However, this cannot be assumed and must be evaluated. For example, see the raltegravir discussion above where accumulation is not predicted from the terminal half-life.

7. The findings in this paper provide a road map for drug formulators to predict the changes in drug absorption needed to yield an extended release dosage form with optimal accumulation and peak to trough ratios at steady-state. At least for some drugs, as demonstrated here, absorption need not be the rate limiting process.

Thanks to a perceptive reviewer who suggests that the sensitivity of $t_{1 / 2 \text {,op }}$ to $k_{\mathrm{a}}$ may be the result of the marked difference between the terminal half life of diazepam $(29.7 \mathrm{~h})$ and its short $t_{1 / 2 \text {,md }}\left(4.10 \mathrm{~h}\right.$, Table IV), since $t_{1 / 2, \mathrm{md}}=0.693 / \mathrm{k}_{10}$ (Eq. 4). Whether this marked difference is diagnostic of drugs amenable to simple formulation of extended release products as described above should be investigated.
Recently there has been a great deal of discussion concerning the ambiguity of the half-life definitions addressed in this manuscript, which can be found on the PharmPK discussion website [http://www.boomer.org/pkin/] under the 2008 discussion sections titled "Terminal half-life and elimination half-life" and "What is 'apparent' half-life?" Further discussions on the web site relate to PK-PD relationships under the topic section "Does half-life in blood inform about drug targets?" There are many drugs where the time course of clinical response does not relate to the pharmacokinetic half-life, such as warfarin, insulin, levodopa, prednisolone, simvastatin, fluoxetine, epoetin and omeprazole among others. However, we contend that under steady-state dosing conditions this lack of a direct relationship between systemic concentrations and effect site concentrations (or measures of clinical response) following a single dose are immaterial if the clinical response is related to systemic exposure.

Another manifestation of the concern about PK/PD discontinuities is found in the anesthesia literature where "context sensitive half-times" are defined (25). The contextsensitive half-time is the time required for systemic concentrations of a drug to decrease by $50 \%$ after discontinuation of drug administration, a value that is a function of the duration of drug administration. Although these anesthesia studies are primarily related to continuous infusions of drug, rather than repeated multiple dose administration, the analysis presented here for intravenous diazepam (Table IVA) is consistent with finding a $50 \%$ fall off time that differs significantly from the terminal half-life.

The lack of continuity of half-life between a pharmacokinetic parameter and a pharmacodynamic effect following a single dose is not the subject of this analysis. A dosage regimen for an i.v. bolus formulation or an immediate release oral formulation will be recommended based on safety and efficacy studies. Daily dose adjustments will result from significant changes in clearance and/or bioavailability. However, dosing interval decisions in many cases will be based on a consideration of half-life. In our experience, clinicians are concerned, especially for narrow therapeutic index drugs, about potential toxicities resulting from peak concentrations that are too high and lack of efficacy for trough concentrations that are too low. Those concerns are addressed by the relationship between dosing interval and the operational halflife. When disease states, age, sex, genetic polymorphisms or alternate dosing formulations change clearance divided by bioavailability, the dosing rate can be appropriately modified to maintain that desired exposure, while calculation of the appropriate $t_{1 / 2 \text {,op }}$ under these conditions can be used to adjust the dosing interval.

\section{CONCLUSIONS}

Half-life is the oldest, but the least well understood pharmacokinetic parameter. The concepts that we have learned about half-life only hold for drugs that following i.v. dosing are best fit by a one-compartment body model, which is true for a very limited number of compounds, if any. Many different half-life terms have been introduced in an attempt to simplify multicompartment kinetics in terms of a single value that would be useful in predicting accumulation at steadystate. These various half-lives have been reviewed here and 
summarized in Table II. To predict drug accumulation upon multiple dosing and peak to trough ratios at steady-state for a particular dosing interval one must determine an operational half-life. For intravenous multiple bolus doses this operational half-life may be approximated by adding a small increment to a half-life related to the mean residence time in the central compartment. For oral multiple doses the operational half-life may only be determined by iterative computer analyses. In addition, this operational half-life may be very sensitive to changes in the absorption rate, and thus drug formulators can use the methodology presented here as a road map in the development of extended release dosage forms.

\section{ACKNOWLEDGEMENTS}

Drs. Sahin and Benet were supported in part during the course of this work by NIH Grant R21 GM75900. The authors appreciate the critical reviews of this work as it progressed and the suggestions of Drs. Malcolm Rowland, Nicholas Holford, Harold Boxenbaum, Svein Øie and Stephen Hwang. Thanks also to Ms. Anita Grover for sharing her preliminary evaluations of everolimus and bepridil simulations with changing absorption rate.

\section{REFERENCES}

1. L. L. Brunton, J. S. Lozo, and K. L. Parker. Goodman and Gilman's The Pharmacological Basis of Therapeutics, 11th edn. McGraw-Hill Medical, New York, NY, 2006.

2. C. F. Lacy, L. L. Armstrong, M. P. Goldman, and L. L. Lance. Drug Information Handbook. A Comprehensive Resource for all Clinicians and Healthcare Professionals, 17th edn. Lexi-Comp, Hudson, OH, 2008-2009.

3. L. Z. Benet. Multiple dosing half-life. A composite term useful in designing therapeutic regimens. Presented at The Third Japanese-American Conference on Pharmacokinetics and Biopharmaceutics, Kyoto, Japan (1985), as available through reference 4.

4. J. G. Wagner. Dosage intervals based on mean residence times. J. Pharm. Sci. 76:35-38 (1987). doi:10.1002/jps.2600760111.

5. P. Veng-Pedersen, and N. B. Modi. Optimal extravascular dosing intervals. J. Pharmacokinet. Biopharm. 19:405-412 (1991). doi:10.1007/BF01061664.

6. K. C. Kwan, N. R. Bohidar, and S. S. Hwang. Estimation of an effective half-life. In L. Z. Benet, G. Levy, and B. Ferraiolo (eds.), Pharmacokinetics: A Modern View, Plenum, New York, NY, 1984, pp. 147-162.

7. H. G. Boxenbaum, and M. Battle. Effective half-life in clinical pharmacology. J. Clin. Pharmacol. 35:763-766 (1995).

8. J. G. Wagner. Drug accumulation. J. Clin. Pharmacol. 7:84-88 (1967).

9. W. A. Colburn. Pharmacokinetic analysis of concentration time data obtained following administration of drugs that are recycled in the bile. J. Pharm. Sci. 73:313-317 (1984). doi:10.1002/ jps.2600730308.

10. A. Hsu, G. R. Granneman, G. Witt, C. Locke, J. Denissen, A. Molla, J. Valdes, J. Smith, K. Erdman, N. Lyons, P. Niu, J.-P. Decourt, J.-B. Fourtillan, J. Girault, and J. M. Leonard.
Multiple-dose pharmacokinetics of ritonavir in human immunodeficiency virus-infected subjects. Antimicrob. Agents Chemother. 41:898-905 (1997).

11. L. Z. Benet. Pharmacokinetics and metabolism of bepridil. Am. J. Cardiol. 55:8C-13C (1985). doi:10.1016/0002-9149(85) 90799-4.

12. P. Augustijns, P. Geusens, and N. Verbeke. Chloroquine levels in blood during chronic treatment of patients with rheumatoid arthritis. Eur. J. Clin. Pharmacol. 42:429-433 (1992).

13. P. Colleste, M. Garle, M. D. Rawlins, and F. Sjoqvist. Interindividual differences in chlorthalidone concentration in plasma and red cells of man after single and multiple doses. Eur. J. Clin. Pharmacol. 9:319-325 (1976). doi:10.1007/ BF00561667.

14. S. A. Kaplan, M. L. Jack, K. Alexander, and R. E. Weinfeld. Pharmacokinetic profile of diazepam in man following single intravenous and oral and chronic oral administrations. J. Pharm. Sci. 16:1789-1796 (1973). doi:10.1002/jps.2600621111.

15. K. Budde, H. H. Neumayer, G. Lehne, M. Winkler, I. A. Hauser, A. Lison, L. Fritsche, J. P. Soulillou, P. Fauchald, J. Dantal, and RADW 102 Renal Transplant Study Group. Tolerability and steady-state pharmacokinetics of everolimus in maintenance renal transplant patients. Nephrol. Dial. Transplant. 19:26062614 (2004). doi:10.1093/ndt/gfh322.

16. D. A. L. Newcombe, F. Bochner, J. M. White, and A. A. Somogyi. Evaluation of levo-alpha-acetylmethdol (LAAM) as an alternative treatment for methadone maintenance patients who regularly experience withdrawal: a pharmacokinetic and pharmacodynamic analysis. Drug Alcohol Depend. 76:63-72 (2004). doi:10.1016/j.drugalcdep.2004.04.004.

17. R. C. Li, P. K. Narang, I. Poggesi, and M. Strolin-Benedetti. A model based assessment of redistribution dependent elimination and bioavailability of rifabutin. Biopharm. Drug Dispos. 17:223236 (1996). doi:10.1002/(SICI)1099-081X(199604)17:3<223::AIDBDD954>3.0.CO;2-S.

18. J. J Zimmerman, and B. D. Kahan. Pharmacokinetics of sirolimus in stable renal transplant patients after multiple oral dose administration. J. Clin. Pharmacol. 37:405-415 (1997).

19. S. Dhillon, and A. Richens. Pharmacokinetics of diazepam in epileptic patients and normal volunteers following intravenous administration. Br. J. Clin. Pharmacol. 12:841-844 (1981).

20. M. Iwamoto, L. A. Wenning, A. S. Petery, M. Laethem, M. De Smet, J. T. Kost, S. A. Merschman, K. M. Strohmaier, S. Ramael, K. C. Lasseter, J. A. Stone, K. M. Gottesdiener, and J. A. Wagner. Safety, tolerability, and pharmacokinetics of raltegravir after single and multiple doses in healthy subjects. Clin. Pharmacol. Ther. 83:293-299 (2008). doi:10.1038/sj. clpt.6100281.

21. J. Boni, J. M. Korth-Bradley, L. S. Richards, S. T. Chiang, D. R. Hicks, and L. Z. Benet. Chiral bioequivalence: effect of absorption rate on racemic etodolac. Clin. Pharmacokinet. 39:459-469 (2000). doi:10.2165/00003088-200039060-00006.

22. P. Macheras, M. Symillides, and C. Repos. An improved intercept method for the assessment of absorption rate in bioequivalence studies. Pharm. Res. 13:1755-1758 (1996). doi:10.1023/A:1016421630290.

23. F. H. Dost. Der Blutspiegel. Thieme, Leipzig, 1953, pp. 252-255.

24. L. Z. Benet. General treatment of linear mammillary models with elimination from any compartment as used in pharmacokinetics. J. Pharm. Sci. 61:536-541 (1972). doi:10.1002/ jps.2600610408.

25. J. M. Bailey. Context-sensitive half-times. What are they and how valuable are they in anaesthesiology. Clin. Pharmacokinet. 41:793-799 (2002). doi:10.2165/00003088-200241110-00001. 